\title{
NEGATIVE TANGENT BUNDLES AND HYPERBOLIC MANIFOLDS
}

\author{
B. WONG
}

ABSTRACT. We construct a family of algebraic manifolds which are hyperbolic in the sense of Kobayashi, but whose tangent bundles are not negative.

\section{Introduction.}

Definition 1. Let $M$ be a complex manifold. The differential Kobayashi metric $K_{M}$ on $M$ is defined as follows:

$$
K_{M}: T(M) \rightarrow R^{+} \cup\{0\} .
$$

Let $(z, v) \in T(M)$; then

$$
K_{M}(z, v)=\inf _{f}\{|t| \mid d f(t)=v, f \in \operatorname{Hol}(D, M), f(0)=z,
$$

$t$ is a tangent vector of $D$ at the origin $\}$,

where $D$ denotes the unit disc, $\operatorname{Hol}(D, M)$ is the family of holomorphic mappings from $D$ to $M$, and $|t|$ is just the Euclidean norm at the origin of $D$.

Definition 2. $M$ is said to be hyperbolic iff $K_{M}$ is nontrivial everywhere on $T(M)$, that is $K_{M}(z, v)=0 \leftrightarrow v=0$, for all $z \in M$ (see Definition 3).

Let $M$ be a compact complex manifold.

Definition 3. The tangent bundle $T(M)$ of $M$ is said to be negative iff $T(M)$ is a strongly pseudo-convex manifold (i.e. admitting a strongly pseudoconvex exhaustion function) whose only compact complex analytic subvariety is the zero section.

A different way to define the negative tangent bundle is: $T(M)$ is negative iff $T^{*}(M)$ (the cotangent bundle) is ample. (See [5] for the definition of ampleness.)

In [4] S. Kobayashi gave a differential geometric proof of the following

THEOREM. Let $M$ be a compact complex manifold whose tangent bundle is negative; then $M$ must be hyperbolic.

A counterexample to the converse of this theorem did not seem to be known. As a consequence of recent results of Brody and Green [1], one can

Received by the editors March 19, 1976.

AMS (MOS) subject classifications (1970). Primary 32H15, 32H20; Secondary 32H25, 32A15.

Key words and phrases. Hyperbolic manifold, negative tangent bundle. 
obtain such an example from their construction. The aim of this note is to point out this interesting fact.

II. A family of algebraic hyperbolic manifolds whose tangent bundles are not negative. In [1], Brody and Green obtained the following remarkable result. (They proved a more general result, but the following description is sufficient for our purpose.)

Theorem (Brody AND GREen). Let $W_{d}=\left\{W_{0}^{d}+W_{1}^{d}+W_{2}^{d}+W_{3}^{d}=0\right\}$ $\subset \mathrm{CP}_{3}$, where $d \geqslant 50$, and is even.

Let us consider a family of hypersurfaces in $\mathrm{CP}_{3}$ given by the following construction:

$$
V_{\varepsilon}=\left\{Z_{0}^{d}+Z_{1}^{d}+Z_{2}^{d}+Z_{3}^{d}+\left(\varepsilon z_{0} z_{1}\right)^{d / 2}+\varepsilon\left(z_{0} z_{2}\right)^{d / 2}=0\right\},
$$

for $\varepsilon$ sufficiently small.

Then those surfaces in $V_{\varepsilon}$ with $\varepsilon \neq 0$ are hyperbolic.

The proof of this theorem depends heavily on the thesis of Robert Brody: C-hyperbolic is equivalent to Kobayashi hyperbolic for compact complex manifold (Harvard, 1975).

We claim that any member of $V_{\varepsilon}$ cannot carry negative tangent bundle. The crucial observation is the following

LemMa. Any hypersurface given by homogeneous polynomial of degree $\geqslant 3$ in $\mathrm{CP}_{3}$ cannot carry negative tangent bundle.

(As a matter of fact, this result is true for any hypersurface given by homogeneous polynomials in $\mathbf{C} P_{n}$ for $n \geqslant 3$, but we just need this lemma to prove our counterexample.

Proof. We need a result of Kleiman [5], which can be stated as follows.

THEOREM. Let $M$ be any nonsingular algebraic surface, and $E$ any ample vector bundle $E$ of rank 2 over $M$. We let $d_{1}, d_{2}$ be the first and second Chern classes of $E$; then the following inequality holds:

$$
\left(d_{1}^{2}-d_{2}\right)(M)>0 .
$$

In our case, we take $T^{*}(M)$ to be $E$.

Note. From our second definition of negative tangent bundle, $T(M)$ is negative $\leftrightarrow T^{*}(M)$ is ample.

We let $C_{1}, C_{2}$ be the first and second Chern classes of $T(M)$; then we have the following relations:

$$
d_{1}=-C_{1}, \quad d_{2}=(-1)^{2} C_{2} .
$$

Note. $\operatorname{dim}_{\mathbf{C}} M=2$.

In order to prove our lemma, one has to show that if $M$ is the hypersurface in our lemma, then $\left(C_{1}^{2}-C_{2}\right)(M)<0$. 
Let $h$ be the first Chern class of the hyperplane bundle $H$ of $\mathrm{CP}_{3}$. By a formula in the appendix of [2], one obtains the following identity:

$$
(1+h)^{4}=\left(1+C_{1}+C_{2}\right)(1+d \cdot h)
$$

(recall $d$ is the degree of $M$ ); then we have

$$
C_{1}=(4-d) \cdot h, \quad C_{2}=\left(6-4 d+d^{2}\right) \cdot h^{2} .
$$

We can easily show

$$
\left(C_{1}^{2}-C_{2}\right)=(10-4 d) \cdot h^{2}
$$

so that we can conclude

$$
\left(C_{1}^{2}-C_{2}\right)(M)<0 \text { if } d \geqslant 3 \text {. }
$$

This completes the proof of the lemma. Q.E.D.

Now we are in the final touch of our assertion. If $\operatorname{dim}_{\mathbf{C}} M=2$, one can see $C_{1}^{2}(M), C_{2}(M)$ are topological invariance. (One way to see this fact is the following: $\left(C_{1}^{2}-2 C_{2}\right)(M)$ is the signature of $M$ if $\operatorname{dim}_{\mathbf{C}} M=2$, and $C_{2}(M)$ is just the Euler characteristic. Both the signature and Euler characteristic are topological invariance, hence imply $C_{1}^{2}(M)$ is also a topological invariance.)

When $\varepsilon=0$, the hypersurface is only the locus of the following homogeneous polynomial:

$$
M_{0}=\left\{Z_{0}^{d}+Z_{1}^{d}+Z_{2}^{d}+Z_{3}^{d}=0\right\}, \quad d \geqslant 50 .
$$

We also observe any member of $V_{\varepsilon}$ is diffeomorphic to $M_{0}$, however by the proof of our lemma, $\left(C_{1}^{2}-C_{2}^{2}\right)\left(M_{0}\right)$ is always less than zero (if $d \geqslant 50$ ), and this number is a topological invariance from previous argument. Therefore we can easily see that any member of $V_{\varepsilon}$ cannot carry a negative tangent bundle. This completes the whole proof.

\section{REFERENCES}

1. Robert Brody and Mark Green, $A$ family of smooth hyperbolic hypersurfaces in $\mathbf{C} P_{3}$. (preprint).

2. F. Hirzebruch, Topological methods in algebraic geometry, 3rd ed., Springer-Verlag, New York, 1966. MR 34 \#2573.

3. S. Kobayashi, Hyperbolic manifolds and holomorphic mappings, Dekker, New York, 1970. MR 43 \#3503.

4. - Negative vector bundles and complex Finsler structures, Nagoya Math. J. 57 (1975).

5. S. Kleiman, Ample vector bundles on algebraic surfaces, Proc. Amer. Math. Soc. 21 (1969), 673-676. MR 40 \#4275.

Department of Mathematics, University of Toronto, Toronto, Ontario M5S 1A7, CANADA 